\title{
Analysis of the friction (spin) welding - preliminary study
}

\author{
Michat Pietrzak ${ }^{1, *}$, Krzysztof Wałęsa ${ }^{1}$, Jan Górecki $^{1}$, and Maciej Berdychowski ${ }^{1}$ \\ ${ }^{1}$ Chair of Basics of Machine Design, Poznan University of Technology, Piotrowo 3, 60-965 Poznań, \\ Poland
}

\begin{abstract}
This article applies to the glue-free bonding of the components made of thermoplastics, specific techniques that use friction heat to plasticize and then join two surfaces. The featured technique is a friction (spin) welding process. Welding parameters were investigated and presented. Spin welding is known to consist of four phases. Analytical calculations were made for the first step. They are focused on the changeability of welding parameters depending on weld velocity. In the next part of this article, calculations including the finite element method were performed. This provided additional data and visualization of the process, which were not available in analytical calculations. Final results of both methods were compared. This made it possible to verify the correctness of theoretical calculations and exactness of FEM analysis.
\end{abstract}

Keywords: vibration welding, friction welding, spin welding, thermoplastics welding

\section{Introduction}

Plastics are known to be easily molded into various shapes in reasonably simple ways [1]. With modern techniques such as injection molding or even rapid technologies allows manufacturing astonishing products in a single step. However, sometimes due to technological processes, an involvement of different materials, sophisticated shapes of components or even production costs, it is required to produce multiple parts and then joining them in rather quick and effective way to fabricate complete component. Moreover, because of the cost of casting forms, there is a growing trend to design parts that are made of standard stock of rods, sheets, tubes, etc [2]. Examples of those solutions are intake manifolds, integrated automobile lights (especially multi-color lenses), or products that are custom made for specific customer demands. Moreover, many manufacturers modify them to meet customer demands, such as adding cleats.

There are many techniques of joining two parts to one another. Some of them require an addition of adhesives (adhesive bonding), others will require using joining parts (mechanical fastening, for example, nuts and bolts), but most desirable methods in the production

*Corresponding author: michal.pietrzak@put.poznan.pl

Reviewers: Michat Bartyś, Milan Sága 
of a single component, are those which make use of material properties without the need of adding another material. Those methods are known as physical bonding, often referred to welding [2].

Plastics, in general, are known to be very good isolators, with very poor heat and electricity transfer [3]. However, friction welding, utilizes those properties and combined with high rate of heat generated at the surface and plasticity in higher temperatures (thermoplastics), leads to a quality joint. Friction welding is a series of friction based solidstate joining processes in which frictional heat is generated due to the rubbing of two surfaces. Usually, one of the components is stationary and second rotates or oscillates (in single or multiple axes). If relative movement is fast enough, the surfaces can be melted and because of poor heat transfer, the following process does not heat up more material than necessary to form a bond [4].

Following method shows many advantages over traditional fusion welding techniques: absence of solidification defects, ability to weld dissimilar materials, lack of consumables, ease of automation, cost-effectiveness and eco-compatibility [4]. Such advantages seem to be very attractive to industry. Moreover, there is a possibility to mold additional ribs or other structural elements to complete component, without much interference into material structure $[1,3,4]$. In many cases, complexity or size of parts could be a challenge for robotic application. It is worth mentioning, that using adhesive can fill small details (for example oil feed) and cause malfunction. An adhesive is also often considered as a weak point in future service scenarios [1]. In opposite, it is said, that this friction welding provides joint nearly as strong as the material itself [5]. Also because bonding proceeds nearly without air in the melting area, it is recommended to use this method for plastics that are vulnerable to oxidize in higher temperatures [1].

To sum up, the joint is established only in a spot where it is designed to be an only between bonding surfaces. It's reasonably cheap and easy to set and provides a good quality connection.

Following article leads off series of articles focused on friction welding, created for better understanding its process.

\subsection{Friction welding techniques and parameters}

As mentioned in the first part of the introduction, friction welding utilizes friction heat to plasticize two rubbing surfaces. In order to plasticize two rubbing surfaces, there must be enough relative speed and they must be pressed against each other. There are two ways to achieve that, therefore there are two main types of friction welding [4]:

- Linear friction welding $(L F W)$ which is based on the reciprocating movement of at least one of the parts (Fig. 1). A process is carried out on specially designed linear friction welders. There are three main welding parameters: weld speed, pressure and process time. An additional parameter is the penetration distance.

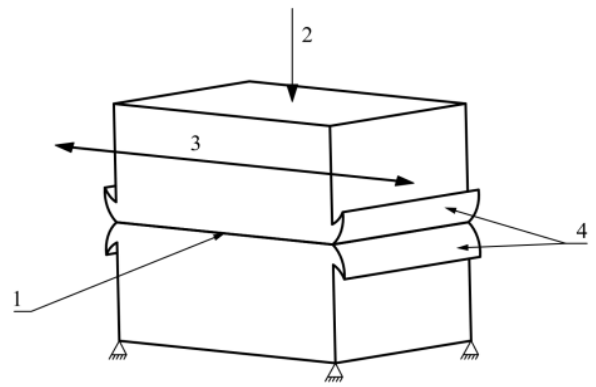

Fig. 1. Linear friction welding. 1 - rubbing surfaces, 2 - pressure, 3 - linear movement, 4 - flash 
Weld speed is commonly divided into amplitude and frequency, as both of them multiplied will give linear velocity. Of course, in more advanced calculations there is a need to consider acceleration of moving part, which will lower the average welding speed.

As mentioned before, another friction welding parameter is pressure. Pressure force is usually not considered as weld parameter, because it will differ with the size of the welding area, whereas pressure does not.

Time of welding process strongly depends on other parameters. In theory, time of welding process is the time that is needed to plasticize, melt and bond two surfaces.

Penetration stands for the sum of shortage in part length. Melted material flows outside welding interface creating flash.

-Rotary friction welding $(R F W)$, in which rubbing between welding surfaces is achieved by rotary movement at least one of the parts (Fig. 2). Usually, those parts have a cylindrical shape - rods or tubes. The following process can be carried on in specially designed friction welders, lathes or even table drillers. To run the RFW process, three main parameters have to be considered: welding speed, pressure and process time. Additional parameters are torque required to maintain desired velocity and penetration.

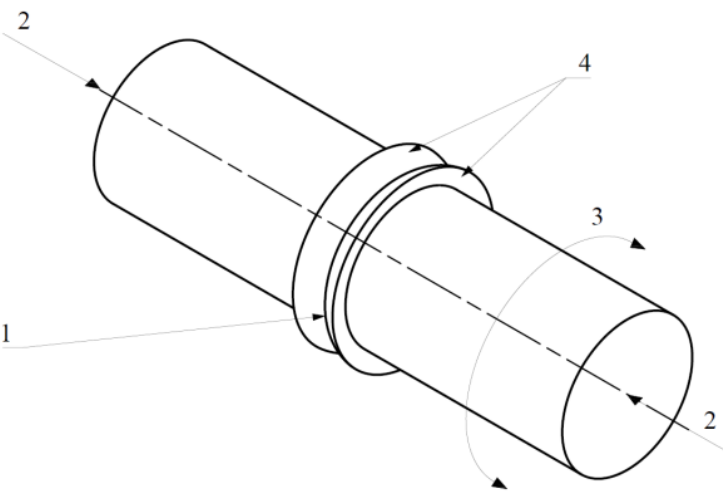

Fig. 2. Rotary friction welding: 1 - rubbing surfaces, 2 - pressure, 3 - welding speed, 4 - flash

In the case of this friction welding method, the welding speed usually refers to relative angular velocity $\omega$. This parameter is often replaced by welding velocity $\mathrm{v}_{0}$, which is relative, linear rubbing speed. This value will be more important in future calculations. However, there is an issue that needs to be explained. As it is commonly known, that linear velocity is a product of multiplying angular velocity and radius. That means, that rubbing speed is different across welding interface (Fig. 3). To overcome this problem, welding parts can be sometimes mounted on eccentricity clamps.

Usually, to simplify calculations, the mean radius can be used (Fig. 3).
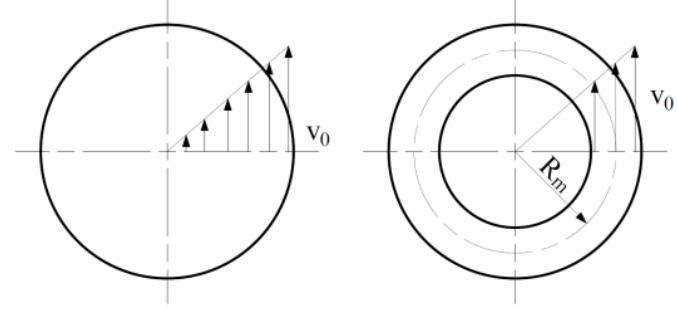

Fig. 3. Difference in welding velocity across a cross section 
Weld pressure, weld time and penetration in rotary friction welding can be described in the same way as for linear friction welding.

\subsection{Phases of friction welding}

Processes presented in Paragraph 1.1 may appear to be very different, but as a matter of fact, they are very similar in their functional principle. Both of them require rubbing velocity, pressure and time to bond two surfaces. In connection with this, both can be described in similar fashion.

Friction welding process consists of four phases. Each one will be shortly described. To make out those phases, it will be useful to present a schematic penetration-time curve (Fig. 4).

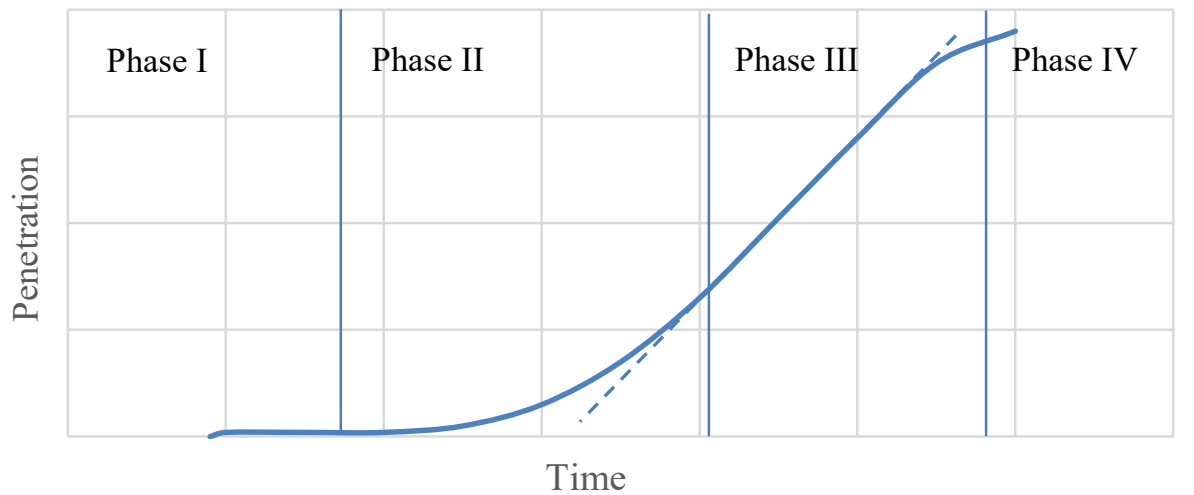

Fig. 4. Phases of friction welding shown on penetration - time graph. The dashed line represents a linear penetration distance increase in time

Phase one, usually named as solid friction, starts when two surfaces start to rub each other. Because of pressure, just after contact penetration increases slightly. During this period, the temperature in the welding area starts to rise, which is caused by Coulomb friction. Generated heat culminates in material, which starts to melt.

Phase two, usually named unsteady evolution of penetration, begins just after material on rubbing surfaces starts to melt. Melted material flows outwards, which results in penetration increase. However, as shown in Fig. 4, penetration is not linear in time. That means, that the rate of melting increases. Molten film thickness increases with time. Eventually, equilibrium is reached at a certain point - a rate at which material is melting is equal to the rate of outflow. After reaching equilibrium between those two ends phase two.

Phase three is usually named as steady penetration phase, because of steady film thickness during this phase. As seen in Fig. 4, penetration is a linear function in time (dashed line). It is said, that this phase is the longest one and has a significant impact on microstructure development, stresses and their orientation at welding spot [1].

Phase four begins just after stopping the relative movement. In this phase, usually named as solidification phase, two elements are joined together.

Following article is focused only on the first part of rotary friction welding.

\section{The first phase of rotary friction welding}

As mentioned before in paragraph 1.2, in the first phase of rotary friction welding, the temperature on weld interfaces rises as a result of Coulomb friction heating. This assumption 
was made due to force measurements during this phase. Results presented in the literature [1] shown, that there is direct proportionality between the shear force and applied normal stress, which is characteristic for Coulomb friction. Moreover, shear force and welding velocity are in phase, which means that there is absent of viscous forces. Those results imply, that Coulomb friction is the only heat generating phenomenon in the first phase of any friction welding. According to the literature [1], shear forces during the first phase can be presented as in (Fig. 5).

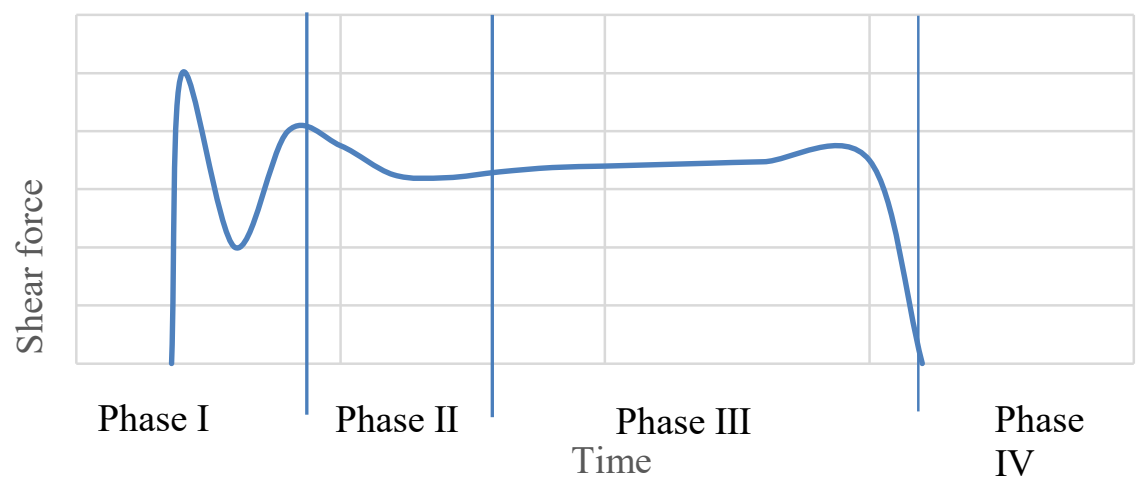

Fig. 5. Phases of friction welding shown on the shear force - time graph

End of phase one is clearly seen in Fig. 5 as a rapid increase in shear force. It is associated with the onset of viscous shear. Interfacial shear forces are also out of phase with the welding velocity, which means that those forces have viscous nature and there is no more Coulomb friction (solid friction).

Also in phase one, there is no penetration other than a very small decrease in element length caused by pressure force.

\subsection{Friction weld phenomenology}

This paragraph is focused on leading out equations for the first phase of rotary friction welding. In order to obtain a model, assumptions had to be made.

The first assumption is that material properties are temperature independent. This will most likely disturb final results, as many attributes are sensitive to the temperature [3]. Lack of verified, temperature dependent data for the investigated polymer is one to blame for the following statement.

The second assumption states that weld speed, weld pressure, and friction coefficient are constant during the first phase ( $\omega=$ const., $p_{0}=$ const., $)$. In real-world conditions, there are always fluctuations of those parameters as shearing forces and material properties changes during the process [3].

The third assumption is the position and geometry of the weld interface in coordination system. Fig. 6 shows the geometry of the model for frictional heating. 


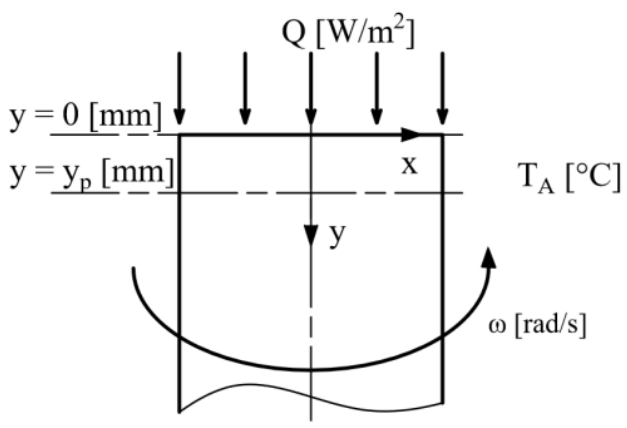

Fig. 6. Geometry of the model of rotary friction welding; $y$ - welding surface, $y_{p}-$ maximum penetrated distance, $\omega$ - welding speed, $\mathrm{T}_{\mathrm{A}}-$ ambient temperature

To clarify, it is worth to add, that weld interface is located on $X Z$ plane with relative rotary motion on $y$-axis. Ambient temperature is specified as $T_{A}$ and is time independent. Welding temperature $(T)$ is more complicated, as it depends on both depth $(y)$ and time. Heating problem is symmetric for both rubbing surfaces, and because of that, it is safe to calculate only one side.

For time $t=0$ and $y \geq 0$ it is stated, that $T=T_{A}$ and at that time process begins. For time $t>0$, surfaces start to rub and heat is generated. It is safe to assume, that highest temperature is located directly on rubbing surfaces.

It is known, that power per square meter at $y=0$ is described by following equation [3]:

$$
Q_{0}=f p_{0} v,
$$

where $: v$ is weld velocity, $f$ is a coefficient of friction and $p_{0}-$ pressure.

However, heat generated between two surfaces heats up two surfaces, therefore for calculating penetration distance or surface temperature for one side, generated heat must be divided by 2 . This equation is useful for steady state heat transfer simulations.

Another problem is to depute the exact temperature of the surface at a given time. Finished equations can be found in [6] - semi-infinite solid, the flux of heat at $y=0$ a prescribed function of the time. It was decided to pass them there in mostly unchanged form. For better understanding, all denotes are stated as follows: $q$ - heat flux, $\lambda$ - thermal conductivity, $t$ - time, $T$ - temperature, $\alpha$ - thermal diffusivity, $f$ - friction coefficient, $p$ - pressure, $v$ - weld velocity.

Derivation starts from a rather simple form, which describes flux per unit time per unit area [6]:

$$
q=-\lambda \frac{\partial T}{\partial y}
$$

It is said, that this flux satisfies the same differential equation as t, namely [6]:

$$
\alpha \frac{\partial^{2} q}{\partial y^{2}}=\frac{\partial q}{\partial t}, y>0, t>0 .
$$

Above equation can be solved with the following assumptions:

$$
\mathrm{q}=Q_{0} \text { constant, } \mathrm{y}=0, \mathrm{t}>0,
$$


and error function:

$$
\begin{gathered}
i^{n} \operatorname{erfcy}=\int_{y}^{\infty} i^{n-1} \operatorname{erfc} \xi d \xi, n=1,2, \ldots, \\
i^{0} \cdot \operatorname{erfc} y=\operatorname{erfc} y, \\
\operatorname{ierfc} y=\frac{1}{\sqrt{\pi}} e^{-y^{2}}-y \cdot \operatorname{erfcy} .
\end{gathered}
$$

Using (4) and (6) for function (3) results as:

$$
q(y, t)=q_{0} \cdot \operatorname{efrc} y \cdot \frac{y}{2 \sqrt{\alpha t}} .
$$

Next, using (5) and (7), there is a possibility to evaluate into:

$$
\begin{aligned}
T(y, t)=\frac{q_{0}}{k} \cdot \int_{y}^{\infty} \operatorname{erfc} \frac{y}{2 \sqrt{\alpha t}} d y & =\frac{2 q_{0} \cdot \sqrt{\alpha t}}{k} \cdot \operatorname{ierfc} \frac{y}{2 \sqrt{\alpha t}}= \\
& =\frac{2 q_{0}}{k}\left\{\left(\frac{\alpha t}{\pi}\right)^{\frac{1}{2}} e^{-\frac{x^{2}}{4 \alpha t}}-\frac{x}{2} \operatorname{erfc} \frac{y}{2 \sqrt{\alpha t}}\right\}
\end{aligned}
$$

In Appendix II for [6] there is a table of values of ierfc function. According to it and (1), the solution of (9) at $y=0$ is given by:

$$
T(y, t)=\frac{2 q_{0}}{k} \cdot\left(\frac{\alpha t}{\pi}\right)^{\frac{1}{2}}=\frac{2 f p_{0} v}{k} \cdot\left(\frac{\alpha t}{\pi}\right)^{\frac{1}{2}} .
$$

Because there are two surfaces that will rub each other, heat absorbed by each surface will be lower than $q_{o}$. To simplify calculations, it was decided, that each surface will absorb $50 \%$ of the heat generated between them $\left(q_{o}\right)$. Therefore, the final form of the equation used in calculating the temperature in a normal direction is presented below:

$$
T(y, t)=\frac{f p_{0} v}{k} \cdot\left(\frac{\alpha t}{\pi}\right)^{\frac{1}{2}}
$$

In order to evaluate duration time of phase I (time to melt rubbing surfaces), equation (11) had to be converted:

$$
t_{I}=\frac{\pi}{\alpha} \cdot\left(\frac{k T_{m}}{f p_{0} v}\right)^{2}
$$

where $T_{m}$ - transition/melting temperature.

There is also a possibility to calculate penetrated distance $\mathrm{y}_{\mathrm{p}}$ :

$$
y_{p}=\left(4 \pi \frac{\lambda T_{m}}{f p_{0} v}\right)^{\frac{1}{2}} .
$$

\section{Theoretical calculation results}

In this paragraph, real values were used in equations from paragraph 2.1. The main goal was to verify dependency of the duration of phase I of pressure. In order to present material characteristics, a Table 1 of physical properties was created [2]. 
Table 1. Physical properties of material

\begin{tabular}{|c|c|c|c|}
\hline Property & Value & Property & Value \\
\hline $\begin{array}{c}\text { Density } \\
\mathrm{g}\left[\frac{\mathrm{g}}{\mathrm{cm}}\right]\end{array}$ & 950 & $\begin{array}{c}\text { Coefficient of friction } \\
\mathrm{f}[-]\end{array}$ & 0.4 \\
\hline $\begin{array}{c}\text { Specific heat } \\
\mathrm{c}\left[\frac{\mathrm{kJ}}{\mathrm{kgK}}\right]\end{array}$ & 1680 & $\begin{array}{c}\text { Melting temperature* } \\
\mathrm{T}_{\mathrm{m}}\left[{ }^{\circ} \mathrm{C}\right]\end{array}$ & 200 \\
\hline $\begin{array}{c}\text { Thermal conductivity } \\
\mathrm{k}\left[\frac{\mathrm{W}}{\mathrm{mK}}\right]\end{array}$ & 0.28 & Poisson's Ratio & 0.42 \\
\hline $\begin{array}{c}\text { Thermal diffusivity } \\
\alpha\left[\frac{\mathrm{mm}}{\mathrm{s}}\right]\end{array}$ & $1.28^{-6}$ & - & - \\
\hline
\end{tabular}

* melting temperature - real temperature gain for ambient temperature $0{ }^{\circ} \mathrm{C}$.

For these particular calculations following geometrical properties were assumed and shown in Fig. 7.

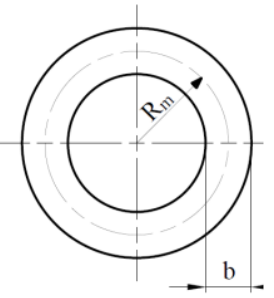

$\mathrm{b}=2 \mathrm{~mm}$

$\mathrm{r}_{\mathrm{m}}=10 \mathrm{~mm}$

$\mathrm{p}_{0}=1 \mathrm{MPa}$

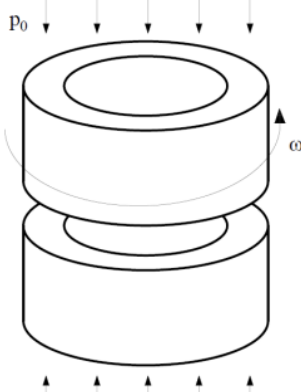

$p_{0}$

Fig. 7. Geometrical properties of welded objects

For better visual presence, results are shown on graphs (Fig. 8-10). The graph on Fig. 8 shows that generated heat is proportional to revolutions per minute. That means, that for example two times higher speed will result in two times higher wattage per square meter.

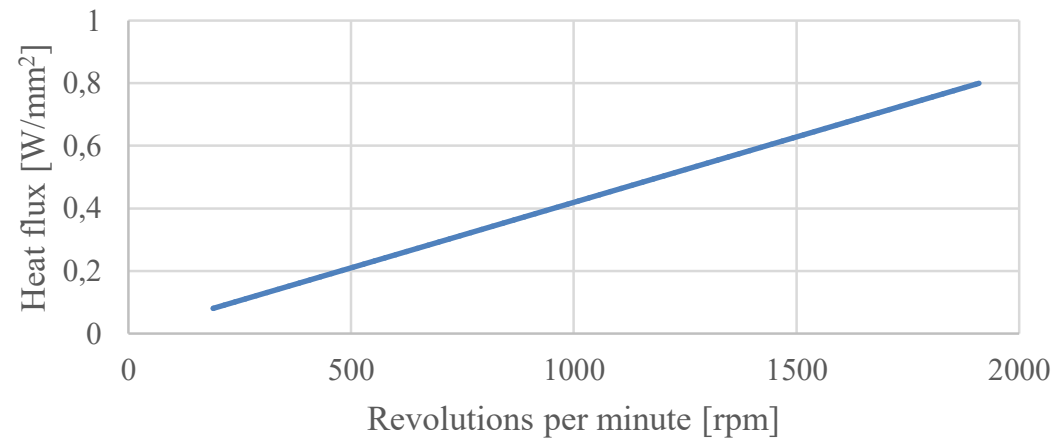

Fig. 8. Heat flux in relation to revolutions per minute 
The graph on Fig. 9 shows, that phase I time decreases with higher welding velocities. As expected - reliance is not linear. It was decided to limit the time of phase I to 10 seconds. Greater amounts of time are not meeting the value of future project requirements of an industrial application. It is worth to notice, that over $500 \mathrm{rpm}$ time decrease is not as sharp as below this mark. It is safe to assume that further MES analyze can be limited to $1000 \mathrm{rpm}$. This will allow to compare the most crucial part of characteristics and shorten analysis time.

Such characteristics are related to generated heat in relation to rotational speed. Higher wattage per square meter will result in shorter heating time.

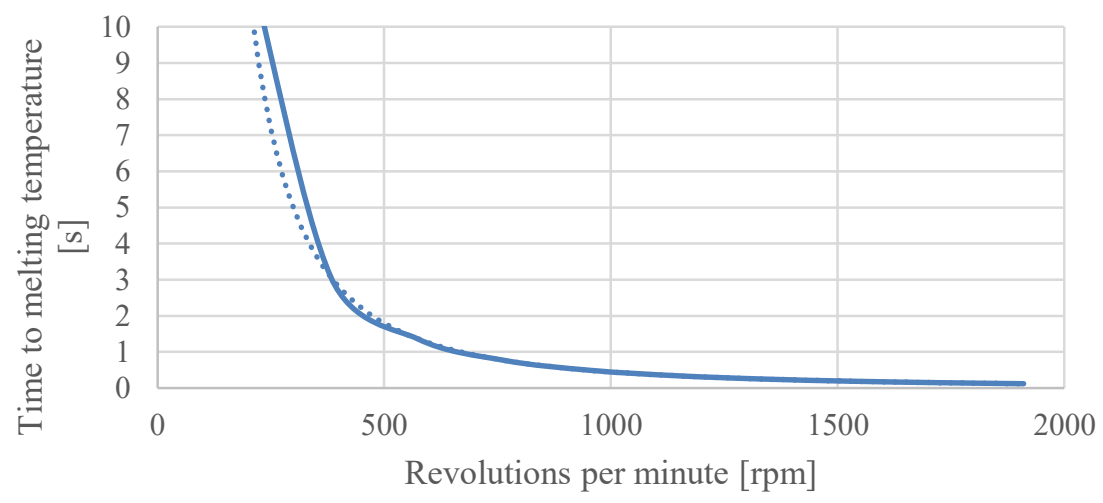

Fig. 9. Time to melting temperature in relation to revolutions per minute

The graph on Fig. 10 shows, that higher weld velocity results in less penetration distance. The decrease of penetrated distance can be clearly seen, but it is not as sharp as a decrease in time. Reason for this behavior is of course heat generated on the surface. Higher heat generation results in faster heating and simply rest of the material, due to poor heat conductivity of the investigated material, will not be able to transfer so much heat/temperature deeper into the material.

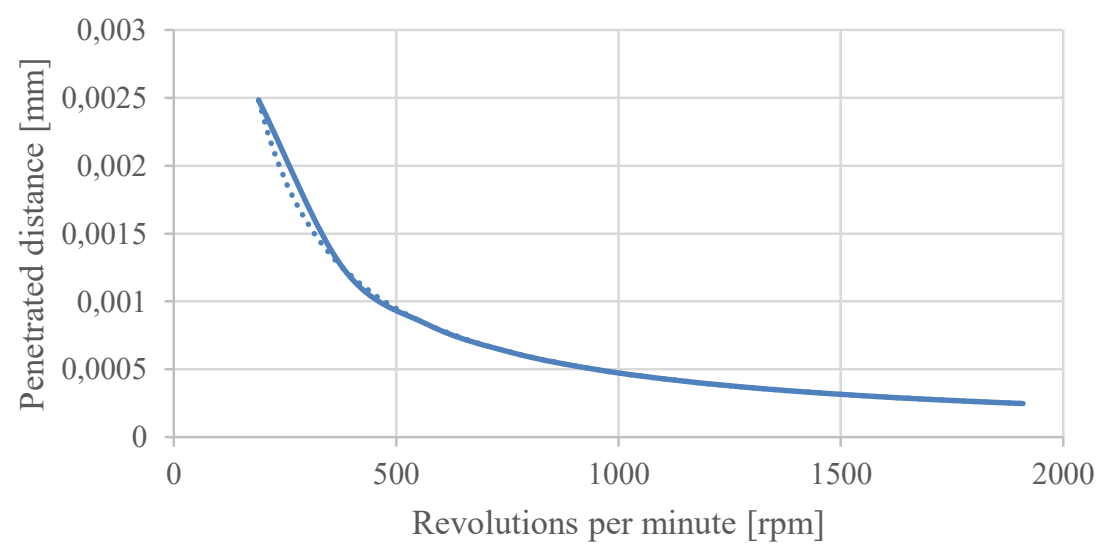

Fig. 10. Penetrated distance in relation to revolutions per minute

\section{Finite element method simulations}


After theoretical calculations, FEM simulations were performed. Material parameters and geometrical properties were carried over from previous calculations (Tab. 1, Fig. 7). Only one parameter was added - height of elements which was set to $3 \mathrm{~mm}$.

Finite Element Method (FEM) is one of the basic methods of computer-aided design. The idea behind this method is to divide one complex system into many simplified systems, and then find a solution by solving successively tasks in smaller component systems. In other words, the assumption of FEM is to divide the continuous model into finite elements that connect at nodes, which creates a discrete geometry model. Thanks to this, the system with an infinite number of degrees of freedom is transformed into a system with a finite number of degrees of freedom.

It was decided to perform simulations in Ansys Workbench, v 19.1. As there is no coupled thermal-displacement analysis available, two different simulations were set - transient thermal and transient structural.

\subsection{Transient thermal analysis (FEM)}

Following analysis was made in order to verify correctness and adjust FEM model used. The secondary goal was to check if it is possible to receive similar results to theoretical calculations. Moreover, it allowed verifying boundary conditions, material properties in the computer environment. Another goal was to prepare the model for subsequent Transient structural type analysis. In order to achieve that, using Transient thermal analysis, heat flux results calculated in paragraph 3 (Fig. 8. Heat flux in relation to revolutions per minute) were applied to rubbing surfaces of two bodies (Fig. 11). Ambient temperature was set to $0^{\circ} \mathrm{C}$, because melting temperature used in paragraph 3, as stated, is temperature increase by the value of $200^{\circ} \mathrm{C}$. For transient-thermal calculations, mesh sizing was set to $1 \mathrm{~mm}$. Sweep method was used.

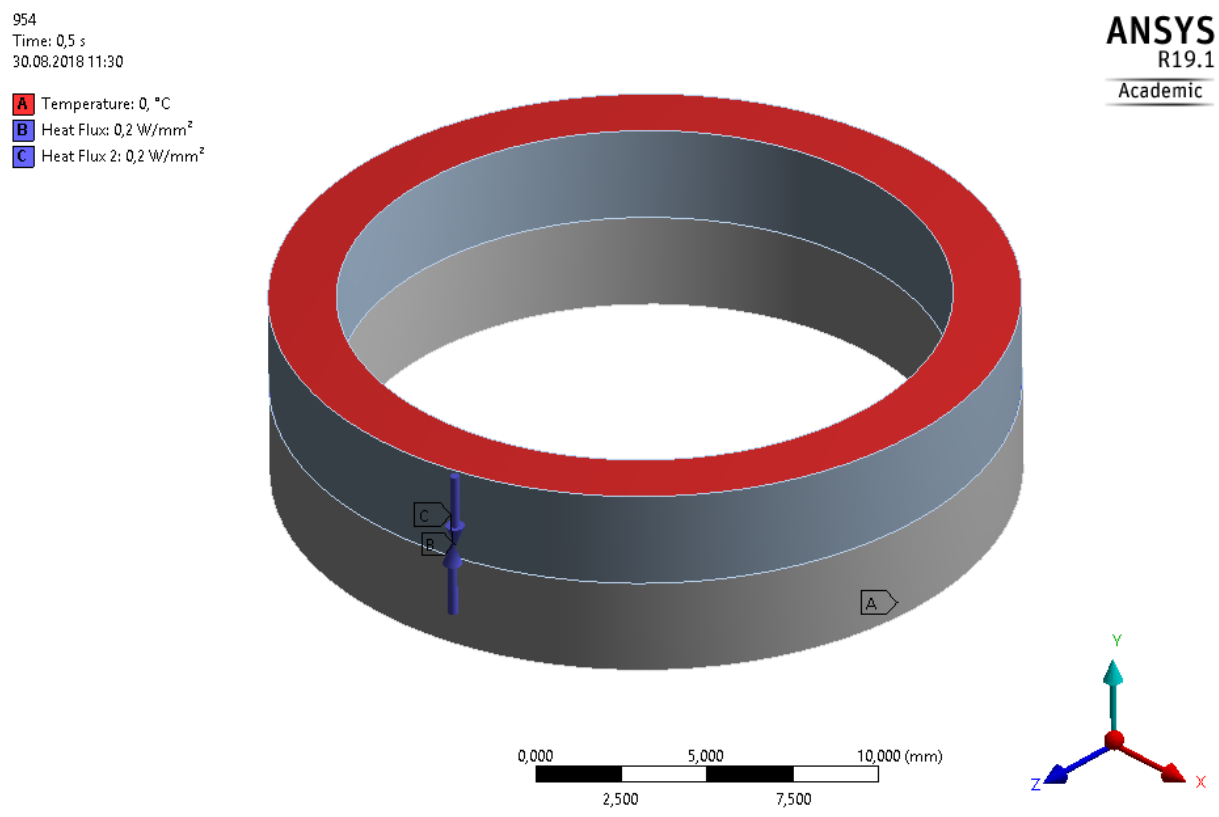

Fig. 11. Model and boundary conditions for the rotation speed of $954 \mathrm{rpm}$ in Transient thermal analysis

Series of analysis were set for heat flux values for the following speeds: $0.2 ; 0.4 ; \ldots ; 2 \mathrm{~m} / \mathrm{s}$ $(190,391.97, \ldots, 1909.86 \mathrm{rpm})$. In order to receive time to melt time, substeps were used in 
analysis settings. Substeps were set to $0.01 \mathrm{~s}$ which means that temperature was updated every single hundredth part of a second. Then, results were exported to Microsoft Excel, where exact time values were calculated. Results are shown in Fig. 13.

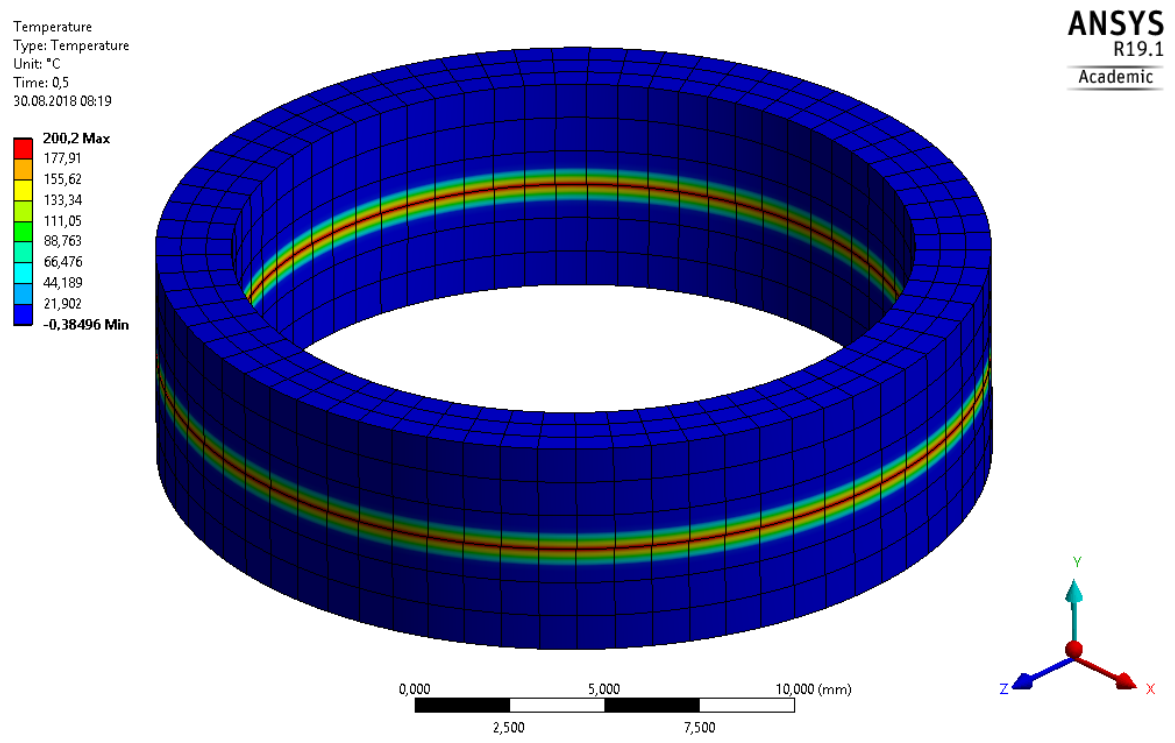

Fig. 12. Results for Transient thermal analysis for the rotation speed of $954 \mathrm{rpm}$

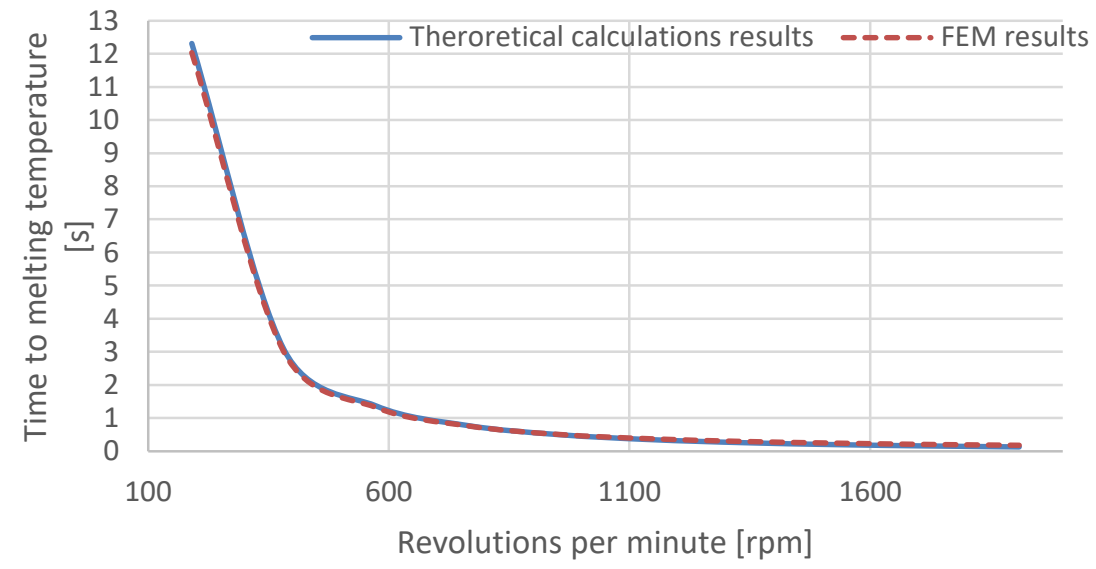

Fig. 13. Results of theoretical calculations juxtaposed with FEM results of the transient thermal analysis

As can be observed in Fig. 13. Results of theoretical calculations juxtaposed with FEM results of the transient thermal analysis, compatibility of previous theoretical calculations and FEM analysis are very similar - in the worst scenario $(1909.86 \mathrm{rpm})$ values are different for $42 \%$ ( $0.05 \mathrm{~s}$ difference). However, up to $1200 \mathrm{rpm}$, overall difference does not exceed $10 \%$. Also, the shape of the curves is very similar. This means, that model, its parameters, mesh, and boundary conditions are matching and it can be used in future analyses. Moreover, conclusions made before (paragraph 3) can be transferred to MES thermal analysis. In addition, temperature results for $954 \mathrm{rpm}$ were added (Fig. 14) 


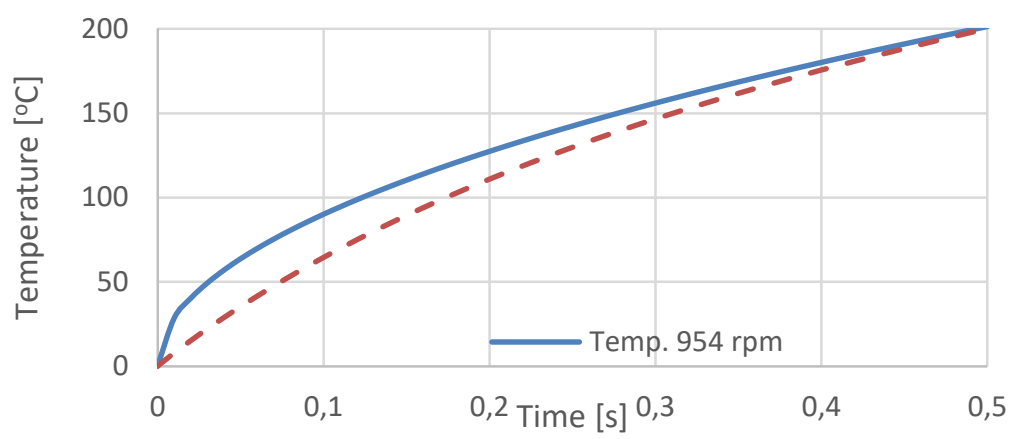

Fig. 14. Temperature increase in time for $954 \mathrm{rpm}$

As seen in Fig. 14, there is over 35\% difference between results at the beginning, but after 0.25 s mark, differences do not exceed $10 \%$.

\section{Conclusions}

Performed calculations have shown that the time of the first phase of friction welding can be approximated by equations written in paragraph 3.1. This can be done only if one agrees to simplify the model by using temperature independent data. The easiest solution to this problem was the FEM analysis.

From transient thermal analysis results provided that are similar to theoretical calculations. That means, that if it is decided to change geometry, material properties or any other of welding parameters, results can be obtained without much effort and will provide additional visualization.

Finite element method analysis will allow much simpler implementation of material parameters that are variable in the function of temperature. In order to accomplish that, the user will only need to insert appropriate data into engineering data tab in Ansys Workbench and run an analysis again.

Obtained model is a solid base for future transient structural analyzes, that will be used to simulate the whole friction welding process. It will be also useful in future studies and research that will lead to the project of the friction welding machine.

\section{References}

1. B. Patham, P. H. Foss, Thermoplastic Vibration Welding: Review of Process Phenomenology and Processing-Structure-Property Interrelationships. (Polymer Engineering and Science, 2011)

2. R. J. Crawford, Y. Tam, Friction welding of plastics. Journal of materials and science $16(1981)$

3. V. K. Stokes, Analysis of the friction (spin)-welding process for thermoplastics. Journal of materials and science 23 (1988)

4. W. Li, S. Shi, F. Wang, Z. Zhang, T. Ma, J. Li, Numerical Simulation of Friction Welding Process Based on ABAQUS Environment. Journal of Engineering Science and Technology Review 5 (3), (2012)

5. H. Potente, Industrial Production Engineering. (1980)

6. H. S. Carslaw, J. C. Jaeger, Conduction of Heat in Solids. Oxford University Press, London (1966) 